\title{
A Regularization Method for the Elliptic Equation with Inhomogeneous Source
}

\author{
Tuan H. Nguyen ${ }^{1}$ and Binh Thanh Tran ${ }^{2}$ \\ ${ }^{1}$ Faculty of Mathematics-Statistics, Ton Duc Thang University, Nguyen Huu Tho Street, District 7, Ho Chi Minh City, Vietnam \\ ${ }^{2}$ Department of Mathematics and Applications, Sai Gon University, 273 An Duong Vuong, District 5, Ho Chi Minh City, Vietnam
}

Correspondence should be addressed to Tuan H. Nguyen; nguyenhuytuan@tdt.edu.vn

Received 31 July 2013; Accepted 3 October 2013; Published 4 March 2014

Academic Editors: F. Colombini and G. L. Karakostas

Copyright (c) 2014 T. H. Nguyen and B. T. Tran. This is an open access article distributed under the Creative Commons Attribution License, which permits unrestricted use, distribution, and reproduction in any medium, provided the original work is properly cited.

We consider the following Cauchy problem for the elliptic equation with inhomogeneous source in a rectangular domain with Dirichlet boundary conditions at $x=0$ and $x=\pi$. The problem is ill-posed. The main aim of this paper is to introduce a regularization method and use it to solve the problem. Some sharp error estimates between the exact solution and its regularization approximation are given and a numerical example shows that the method works effectively.

\section{Introduction}

The Cauchy problem for the elliptic equation has been extensively investigated in many practical areas. For example, some problems relating to geophysics [1], plasma physics [2], and bioelectric field problems [3] are equivalent to solving the Cauchy problem for the elliptic equation. In this paper, we consider the following Cauchy problem for elliptic equation with nonhomogeneous source:

$$
\begin{gathered}
u_{x x}+u_{y y}=f(x, y), \quad(x, y) \in(0, \pi) \times(0,1), \\
u(0, y)=u(\pi, y)=0, \\
u_{y}(x, 0)=0, \\
u(x, 0)=g(x),
\end{gathered}
$$

where $g \in L^{2}(0, \pi), f \in L^{2}\left(0,1 ; L^{2}(0, \pi)\right)$ are given.

Problem (1)-(4) is well known to be ill-posed in the sense of Hadamard: a small perturbation in the data $g$ may cause dramatically large errors in the solution $u(x, y)$ for $0<y \leq 1$. An explicit example to emphasize this fact is given in [4]. In the past, there were many studies on the homogeneous problem, that is $f=0$ in (1). Using the boundary element method, the homogeneous problems were considered in [5-7] and the references therein. Similarly, many authors have investigated the Cauchy problem for linear homogeneous elliptic equation, for example, the quasireversibility method [4, 8-10], fourth-order modified method [11, 12], Fourier truncation regularized method (or spectral regularized method) [13-15], the Backus-Gilbert algorithm [16] and so forth. Some other authors also considered the homogeneous problem such as Beskos [5], Eldén et al. [17, 18], Marin and Lesnic [19], Qin and Wei [20], Regińska and Tautenhahn [21], Tautenhahn [22].

Very recently, in 2009, Hào and his group [23] applied the nonlocal-boundary value method to regularize the abstract homogeneous elliptic problem. This method is also given in [24] for solving an elliptic problem with homogeneous source in a cylindrical domain. A mollification regularization method for the Cauchy problem in a multidimensional case has been considered in the recent paper of Cheng and his group [25].

Although there are many papers on the homogeneous elliptic equation, the result on the inhomogeneous case is very scarce, while the inhomogeneous case is, of course, more general and nearer to practical application than the homogeneous one. Shortly, it allows the occurrence of some elliptic source which is inevitable in nature. The main aim of this 
paper is to present a simple and effective regularization method and investigate the error estimate between the regularization solution and the exact solution. In a sense, this paper may be an extension of many previous results.

The remainder of the paper is divided into two sections. In Section 2, we will study the regularization of problem (1)-(4) and obtain convergence estimates. In Section 3, a numerical test case for inhomogeneous problems is given to describe the effectiveness of our method.

\section{Regularization and Error Estimate}

By the method of separation of variables, the solution of problem (1)-(4) is given by

$$
\begin{aligned}
u(x, y)=\sum_{n=1}^{\infty}[ & \left(\frac{e^{n y}+e^{-n y}}{2}\right) g_{n} \\
& \left.+\int_{0}^{y}\left(\frac{e^{n(y-s)}-e^{n(s-y)}}{2 n}\right) f_{n}(s) d s\right] \sin n x
\end{aligned}
$$

where

$$
\begin{aligned}
g_{n} & =\frac{2}{\pi} \int_{0}^{\pi} g(x) \sin n x d x, \\
f_{n}(s) & =\frac{2}{\pi} \int_{0}^{\pi} f(x, s) \sin n x d x .
\end{aligned}
$$

We can see that the instability is caused by the fast growth of $e^{n y}, y>0$ as $n$ tends to infinity. Even though these exact Fourier coefficients $g_{n}, f_{n}(s)$ may tend to zero rapidly, in practice, performing classical calculation is impossible because the given data is usually diffused by a variety of reasons such as round-off error and measurement error. A small perturbation in the data can arbitrarily deduce a large error in the solution. Therefore, some special regularization methods are required. From (5), we replace the term $e^{n y}$ that causes dramatically the increasing of the right side by several bounded approximations. We assume that the exact data $g(x)$ and the measured data $g^{\epsilon}(x)$ both belong to $L^{2}(0, \pi)$ and satisfy $\left\|g^{\epsilon}-g\right\|_{2} \leq \epsilon$ where $\|\cdot\|_{2}$ is the norm on $L^{2}(0, \pi)$ and $\epsilon$ denotes the noise level, respectively.

In the paper, we will use a modification method to regularize our problem. The regularized solution is given as follows:

$$
\begin{aligned}
& u^{\epsilon}(x, y) \\
& =\sum_{n=1}^{\infty}\left[\frac{1}{2}\left(\frac{1}{\alpha+e^{-n y}}+e^{-n y}\right) g_{n}\right. \\
& \left.\quad+\int_{0}^{y} \frac{1}{2 n}\left(\frac{e^{-n s}}{\alpha+e^{-n y}}-e^{n(s-y)}\right) f_{n}(s) d s\right] \sin n x .
\end{aligned}
$$

Here $\alpha \in(0,1)$ is a parameter regularization which depends on $\epsilon$. The explicit error estimates including error estimates have been given according to some priori assumptions on the regularity of the exact solution.

Let $v^{\epsilon}$ be the solution of problem (7) corresponding to the measured data $g^{\epsilon}$. Then, it is given by

$$
\begin{aligned}
& v^{\epsilon}(x, y) \\
& =\sum_{n=1}^{\infty}\left[\frac{1}{2}\left(\frac{1}{\alpha+e^{-n y}}+e^{-n y}\right) g_{n}^{\epsilon}\right. \\
& \left.\quad+\int_{0}^{y} \frac{1}{2 n}\left(\frac{e^{-n s}}{\alpha+e^{-n y}}-e^{n(s-y)}\right) f_{n}(s) d s\right] \sin n x
\end{aligned}
$$

We first have the following theorem.

Theorem 1. Let $g, g^{\epsilon} \in L^{2}(0, \pi)$ such that $\left\|g^{\epsilon}-g\right\|_{2} \leq \epsilon$. Then one has

$$
\left\|v^{\epsilon}(\cdot, y)-u^{\epsilon}(\cdot, y)\right\|_{2} \leq \alpha^{-1} \epsilon,
$$

for all $y \in[0,1]$.

Proof. It follows from (7) and (8) that

$$
\begin{aligned}
& v^{\epsilon}(\cdot, y)-u^{\epsilon}(\cdot, y) \\
& \quad=\sum_{n=1}^{\infty}\left[\frac{1}{2}\left(\frac{1}{\alpha+e^{-n y}}+e^{-n y}\right)\left(g_{n}^{\epsilon}-g_{n}\right)\right] \sin n x .
\end{aligned}
$$

We have

$$
\begin{aligned}
\left\|v^{\epsilon}(\cdot, y)-u^{\epsilon}(\cdot, y)\right\|_{2}^{2} & =\frac{\pi}{2} \sum_{n=1}^{\infty} \frac{1}{4}\left(\frac{1}{\alpha+e^{-n y}}+e^{-n y}\right)^{2}\left|g_{n}^{\epsilon}-g_{n}\right|^{2} \\
& \leq \frac{\pi}{8} \sum_{n=1}^{\infty}\left(\frac{1}{\alpha}+1\right)^{2}\left|g_{n}^{\epsilon}-g_{n}\right|^{2} \\
& \leq \alpha^{-2}\left\|g^{\epsilon}-g\right\|_{2}^{2} \leq \alpha^{-2} \epsilon^{2} .
\end{aligned}
$$

Therefore, we get

$$
\left\|v^{\epsilon}(\cdot, y)-u^{\epsilon}(\cdot, y)\right\|_{2} \leq \alpha^{-1} \epsilon .
$$

This completes the proof of Theorem 1.

Theorem 2. Let $g, g^{\epsilon}$ be as in Theorem 1. Assume that $\int_{0}^{1} \sum_{n=1}^{\infty} e^{2 n} f_{n}^{2}(s) d s<\infty$. If we select $\alpha=\epsilon^{1 / 2}$, then for every $y \in[0,1]$ one has

$$
\left\|v^{\epsilon}(\cdot, y)-u(\cdot, y)\right\|_{2} \leq M \epsilon^{(1-y) / 2},
$$

where

$$
\begin{aligned}
M= & +\frac{\sqrt{3}}{2} \\
& \times \sqrt{\|u(\cdot, 1)\|_{2}^{2}+\left\|u_{y}(\cdot, 1)\right\|_{2}^{2}+\frac{\pi}{2} \int_{0}^{1} \sum_{n=1}^{\infty} e^{2 n} f_{n}^{2}(s) d s .}
\end{aligned}
$$


ISRN Mathematical Analysis

3

Proof. First, we have

$$
\begin{gathered}
u_{n}(y)=\left(\frac{e^{n y}+e^{-n y}}{2}\right) g_{n}+\int_{0}^{y}\left(\frac{e^{n(y-s)}-e^{n(s-y)}}{2 n}\right) f_{n}(s) d s, \\
u_{n}^{\epsilon}(y)=\frac{1}{2}\left(\frac{1}{\alpha+e^{-n y}}+e^{-n y}\right) g_{n} \\
+\int_{0}^{y} \frac{1}{2 n}\left(\frac{e^{-n s}}{\alpha+e^{-n y}}-e^{n(s-y)}\right) f_{n}(s) d s .
\end{gathered}
$$

Subtracting (16) to (15), we have

$$
\begin{aligned}
u_{n}^{\epsilon}(y) & -u_{n}(y) \\
= & \frac{1}{2}\left(\frac{1}{\alpha+e^{-n y}}-e^{n y}\right) g_{n} \\
& +\frac{1}{2 n} \int_{0}^{y}\left(\frac{e^{-n s}}{\alpha+e^{-n y}}-e^{n(y-s)}\right) f_{n}(s) d s \\
= & \frac{-1}{2}\left(\frac{\alpha}{\alpha+e^{-n y}}\right)\left[e^{n y} g_{n}+\frac{1}{n} \int_{0}^{y} e^{n(y-s)} f_{n}(s) d s\right] .
\end{aligned}
$$

We have

$$
\begin{aligned}
& u_{y}(x, y) \\
& =\sum_{n=1}^{\infty}\left[n\left(\frac{e^{n y}-e^{-n y}}{2}\right) g_{n}\right. \\
& \left.\quad+\int_{0}^{y} n\left(\frac{e^{n(y-s)}+e^{n(s-y)}}{2 n}\right) f_{n}(s) d s\right] \sin n x \\
& =\sum_{n=1}^{\infty} n\left[\left(\frac{e^{n y}-e^{-n y}}{2}\right) g_{n}\right. \\
& \left.\quad+\int_{0}^{y}\left(\frac{e^{n(y-s)}+e^{n(s-y)}}{2 n}\right) f_{n}(s) d s\right] \sin n x .
\end{aligned}
$$

From (15), we have

$$
\begin{aligned}
\frac{1}{n} \frac{d}{d y} u_{n}(y)= & \left(\frac{e^{n y}-e^{-n y}}{2}\right) g_{n} \\
& +\int_{0}^{y}\left(\frac{e^{n(y-s)}+e^{n(s-y)}}{2 n}\right) f_{n}(s) d s .
\end{aligned}
$$

Combining (15) and (19), we get

$$
u_{n}(y)+\frac{1}{n} \frac{d}{d y} u_{n}(y)=e^{n y} g_{n}+\frac{1}{n} \int_{0}^{y} e^{n(y-s)} f_{n}(s) d s .
$$

Let $y=1$; we have

$$
\begin{aligned}
u_{n}(1)+\frac{1}{n} \frac{d}{d y} u_{n}(1) & =e^{n} g_{n}+\frac{1}{n} \int_{0}^{1} e^{n(1-s)} f_{n}(s) d s \\
& =e^{n}\left[g_{n}+\frac{1}{n} \int_{0}^{1} e^{-n s} f_{n}(s) d s\right] .
\end{aligned}
$$

Therefore, we get

$$
g_{n}=e^{-n}\left[u_{n}(1)+\frac{1}{n} \frac{d}{d y} u_{n}(1)\right]-\frac{1}{n} \int_{0}^{1} e^{-n s} f_{n}(s) d s .
$$

From (17) and (22), we have

$$
\begin{aligned}
& \left|u_{n}^{\epsilon}(y)-u_{n}(y)\right| \\
& =\frac{1}{2}\left(\frac{\alpha}{\alpha+e^{-n y}}\right) \mid e^{n y}\left[e^{-n} u_{n}(1)+\frac{e^{-n}}{n} \frac{d}{d y} u_{n}(1)\right. \\
& \left.-\frac{1}{n} \int_{0}^{1} e^{-n s} f_{n}(s) d s\right] \\
& +\frac{1}{n} \int_{0}^{y} e^{n(y-s)} f_{n}(s) d s \mid \\
& =\frac{1}{2}\left(\frac{\alpha}{\alpha+e^{-n y}}\right) \mid e^{n(y-1)}\left[u_{n}(1)+\frac{1}{n} \frac{d}{d y} u_{n}(1)\right] \\
& -\frac{1}{n} \int_{y}^{1} e^{n(y-s)} f_{n}(s) d s \\
& \leq \frac{1}{2} \alpha\left(\frac{e^{-n(1-y)}}{\alpha+e^{-n}}\right)\left[\left|u_{n}(1)\right|+\left|\frac{d}{d y} u_{n}(1)\right|\right. \\
& \left.+\int_{0}^{1}\left|e^{n} f_{n}(s)\right| d s\right] \text {. }
\end{aligned}
$$

Moreover, one has, for $\tau>t>0$ and $\alpha>0$,

$$
\frac{e^{-t n}}{\alpha+e^{-\tau n}}=\frac{1}{\left(\alpha e^{\tau n}+1\right)^{t / \tau}\left(\alpha+e^{-\tau n}\right)^{1-t / \tau}} \leq \alpha^{t / \tau-1} .
$$

Letting $\tau=1, t=1-y$, we get

$$
\frac{e^{-n(1-y)}}{\alpha+e^{-n}} \leq \alpha^{-y}
$$

From (23), (25), we have

$$
\begin{aligned}
& \left|u_{n}^{\epsilon}(y)-u_{n}(y)\right| \\
& \quad \leq \frac{1}{2} \alpha^{1-y}\left[\left|u_{n}(1)\right|+\left|\frac{d}{d y} u_{n}(1)\right|+\int_{0}^{1}\left|e^{n} f_{n}(s)\right| d s\right] .
\end{aligned}
$$

Applying the inequality $(a+b+c)^{2} \leq 3\left(a^{2}+b^{2}+c^{2}\right)$, we have

$$
\begin{aligned}
& \left|u_{n}^{\epsilon}(y)-u_{n}(y)\right|^{2} \\
& \leq \frac{3}{4} \alpha^{2(1-y)}\left[\left|u_{n}(1)\right|^{2}\right. \\
& \left.\quad+\left|\frac{d}{d y} u_{n}(1)\right|^{2}+\left(\int_{0}^{1}\left|e^{n} f_{n}(s)\right| d s\right)^{2}\right] \\
& \leq \frac{3}{4} \alpha^{2(1-y)}\left[\left|u_{n}(1)\right|^{2}\right. \\
& \left.\quad+\left|\frac{d}{d y} u_{n}(1)\right|^{2}+\int_{0}^{1} e^{2 n} f_{n}^{2}(s) d s\right] .
\end{aligned}
$$


Thus

$$
\begin{aligned}
& \left\|u^{\epsilon}(\cdot, y)-u(\cdot, y)\right\|_{2}^{2} \\
& =\frac{\pi}{2} \sum_{n=1}^{\infty}\left|u_{n}^{\epsilon}(y)-u_{n}(y)\right|^{2} \\
& \leq \frac{3}{4} \alpha^{2(1-y)}\left(\frac{\pi}{2} \sum_{n=1}^{\infty}\left|u_{n}(1)\right|^{2}\right. \\
& +\frac{\pi}{2} \sum_{n=1}^{\infty}\left|\frac{d}{d y} u_{n}(1)\right|^{2} \\
& \left.\quad+\frac{\pi}{2} \sum_{n=1}^{\infty} \int_{0}^{1} e^{2 n} f_{n}^{2}(s) d s\right) \\
& \quad+\frac{3}{4}(\cdot, 1)\left\|_{2}^{2}+\right\| u_{y}(\cdot, 1) \|_{2}^{2} \\
& \left.\quad+\frac{\pi}{2} \int_{0}^{1} \sum_{n=1}^{\infty} e^{2 n} f_{n}^{2}(s) d s\right),
\end{aligned}
$$

or we get

$$
\begin{aligned}
& \left\|u^{\epsilon}(\cdot, y)-u(\cdot, y)\right\|_{2} \\
& \leq \frac{\sqrt{3}}{2} \alpha^{1-y} \sqrt{\|u(\cdot, 1)\|_{2}^{2}+\left\|u_{y}(\cdot, 1)\right\|_{2}^{2}+\frac{\pi}{2} \int_{0}^{1} \sum_{n=1}^{\infty} e^{2 n} f_{n}^{2}(s) d s .}
\end{aligned}
$$

Using Theorem 1 and (29), we get

$$
\begin{aligned}
& \left\|v^{\epsilon}(\cdot, y)-u(\cdot, y)\right\|_{2} \\
& \leq\left\|v^{\epsilon}(\cdot, y)-u^{\epsilon}(\cdot, y)\right\|_{2}+\left\|u^{\epsilon}(\cdot, y)-u(\cdot, y)\right\|_{2} \\
& \leq \alpha^{-1} \epsilon \\
& +\frac{\sqrt{3}}{2} \alpha^{1-y} \sqrt{\|u(\cdot, 1)\|_{2}^{2}+\left\|u_{y}(\cdot, 1)\right\|_{2}^{2}+\frac{\pi}{2} \int_{0}^{1} \sum_{n=1}^{\infty} e^{2 n} f_{n}^{2}(s) d s} \\
& =\epsilon^{1 / 2} \\
& +\frac{\sqrt{3}}{2} \epsilon^{(1-y) / 2} \sqrt{\|u(\cdot, 1)\|_{2}^{2}+\left\|u_{y}(\cdot, 1)\right\|_{2}^{2}+\frac{\pi}{2} \int_{0}^{1} \sum_{n=1}^{\infty} e^{2 n} f_{n}^{2}(s) d s} \\
& \leq M \epsilon^{(1-y) / 2},
\end{aligned}
$$

where

$$
\begin{aligned}
M=1 & +\frac{\sqrt{3}}{2} \\
& \times \sqrt{\|u(\cdot, 1)\|_{2}^{2}+\left\|u_{y}(\cdot, 1)\right\|_{2}^{2}+\frac{\pi}{2} \int_{0}^{1} \sum_{n=1}^{\infty} e^{2 n} f_{n}^{2}(s) d s .}
\end{aligned}
$$

Remark 3. From (13), as $y \rightarrow 1$, the accuracy of regularized solution becomes progressively lower. To retain the continuous dependence of the solution at $y=1$, we introduce the following theorem.

Theorem 4. Assume that problem (1)-(4) has a solution u such that $u_{y} \in L^{2}\left((0,1) ; L^{2}(0, \pi)\right)$ and

$$
\int_{0}^{1} \sum_{n=1}^{\infty} e^{2 n} f_{n}^{2}(s) d s<\infty .
$$

Then for all $\epsilon \in(0,1)$ there exists a $y_{\epsilon}>0$ such that

$$
\left\|u^{\epsilon}\left(\cdot, y_{\epsilon}\right)-u(\cdot, 1)\right\|_{2} \leq 2 C_{1}\left(\ln \left(\frac{1}{\epsilon}\right)\right)^{-1 / 4}
$$

where

$$
N=\sqrt{\int_{0}^{1}\left\|u_{y}(\cdot, s)\right\|_{2}^{2} d s}
$$

$C_{1}$

$$
\begin{aligned}
=\max & \left\{N, \frac{\sqrt{3}}{2}\right. \\
& \times \sqrt{\left.\|u(\cdot, 1)\|_{2}^{2}+\left\|u_{y}(\cdot, 1)\right\|_{2}^{2}+\frac{\pi}{2} \int_{0}^{1} \sum_{n=1}^{\infty} e^{2 n} f_{n}^{2}(s) d s\right\} .} .
\end{aligned}
$$

Proof. We have

$$
u(x, 1)-u(x, y)=\int_{y}^{1} u_{y}(x, s) d s
$$

It follows that

$$
\|u(\cdot, y)-u(\cdot, 1)\|_{2}^{2} \leq(1-y) \int_{y}^{1}\left\|u_{y}(\cdot, s)\right\|_{2}^{2} d s=N^{2}(1-y) .
$$

Using (29), noticing that $\alpha=\epsilon^{1 / 2}$, and (34), we have

$$
\begin{aligned}
\left\|u^{\epsilon}(\cdot, y)-u(\cdot, 1)\right\|_{2} \leq & \left\|u^{\epsilon}(\cdot, y)-u(\cdot, y)\right\|_{2} \\
& +\|u(\cdot, y)-u(\cdot, 1)\|_{2} \\
\leq & C_{1}\left(\sqrt{1-y}+\epsilon^{(1-y) / 2}\right) .
\end{aligned}
$$

For every $\epsilon \in(0,1)$, there exists uniquely a positive number $y_{\epsilon}$ such that $\sqrt{1-y_{\epsilon}}=\epsilon^{\left(1-y_{\epsilon}\right) / 2}$; that is

$$
\frac{\ln \left(1-y_{\epsilon}\right)}{1-y_{\epsilon}}=\ln \epsilon
$$

Using inequality $\ln (1-y)>-1 /(1-y)$ for every $0<y<1$, we get

$$
\left\|u^{\epsilon}\left(\cdot, y_{\epsilon}\right)-u(\cdot, 1)\right\|_{2} \leq 2 C_{1}\left(\ln \left(\frac{1}{\epsilon}\right)\right)^{-1 / 4} .
$$

This completes the proof of Theorem 4 . 
Theorem 5. Let $g, g^{\epsilon}, u$ be as in Theorem 4 and (32) holds. Then one can construct a function $w^{\epsilon}$ satisfying

$$
\left\|w^{\epsilon}(\cdot, y)-u(\cdot, y)\right\|_{2} \leq M \epsilon^{(1-y) / 2}
$$

for every $y \in(0,1)$ and

$$
\left\|w^{\epsilon}(\cdot, 1)-u(\cdot, 1)\right\|_{2} \leq C\left(\ln \left(\frac{1}{\epsilon}\right)\right)^{-1 / 4}
$$

where

$$
\begin{aligned}
M=2 & +\frac{\sqrt{3}}{2} \\
& \times \sqrt{\|u(\cdot, 1)\|_{2}^{2}+\left\|u_{y}(\cdot, 1)\right\|_{2}^{2}+\frac{\pi}{2} \int_{0}^{1} \sum_{n=1}^{\infty} e^{2 n} f_{n}^{2}(s) d s,} \\
C_{1} \max & \left\{\sqrt{\int_{0}^{1}\left\|u_{y}(\cdot, s)\right\|_{2}^{2} d s, \frac{\sqrt{3}}{2}}\right. \\
& \left.\times \sqrt{\left.\|u(\cdot, 1)\|_{2}^{2}+\left\|u_{y}(\cdot, 1)\right\|_{2}^{2}+\frac{\pi}{2} \int_{0}^{1} \sum_{n=1}^{\infty} e^{2 n} f_{n}^{2}(s) d s\right\}}\right\}
\end{aligned}
$$

Proof. Let $y_{\epsilon}$ be the unique solution of

$$
\sqrt{1-y_{\epsilon}}=\epsilon^{\left(1-y_{\epsilon}\right) / 2} \text {. }
$$

We define a function $w^{\epsilon}$ as follows:

$$
w^{\epsilon}(\cdot, y)= \begin{cases}v^{\epsilon}(\cdot, y), & 0<y<1, \\ v^{\epsilon}\left(\cdot, y_{\epsilon}\right), & y=1 .\end{cases}
$$

From Theorem 1, we have

$$
\begin{aligned}
\left\|w^{\epsilon}(\cdot, y)-u(\cdot, y)\right\|_{2} & =\left\|v^{\epsilon}(\cdot, y)-u(\cdot, y)\right\|_{2} \\
& \leq M \epsilon^{(1-y) / 2}
\end{aligned}
$$

for every $y \in(0,1)$. From Theorem 2 , we have

$$
\left\|u^{\epsilon}\left(\cdot, y_{\epsilon}\right)-u(\cdot, 1)\right\|_{2} \leq 2 C_{1}\left(\ln \left(\frac{1}{\epsilon}\right)\right)^{-1 / 4}
$$

Using Theorem 1, noticing that $\alpha=\epsilon^{1 / 2},(43)$, and (46), we get

$$
\begin{aligned}
\left\|w^{\epsilon}(\cdot, 1)-u(\cdot, 1)\right\|_{2} & =\left\|v^{\epsilon}\left(\cdot, y_{\epsilon}\right)-u(\cdot, 1)\right\|_{2} \\
\leq & \left\|v^{\epsilon}\left(\cdot, y_{\epsilon}\right)-u^{\epsilon}\left(\cdot, y_{\epsilon}\right)\right\|_{2} \\
& +\left\|u^{\epsilon}\left(\cdot, y_{\epsilon}\right)-u(\cdot, 1)\right\|_{2} \\
\leq & \epsilon^{1 / 2}+\epsilon+2 C_{1}\left(\ln \left(\frac{1}{\epsilon}\right)\right)^{-1 / 4} \\
\leq & C\left(\ln \left(\frac{1}{\epsilon}\right)\right)^{-1 / 4}
\end{aligned}
$$

where

$$
C=2+2 C_{1} \text {. }
$$

This completes the proof of Theorem 5 .

Remark 6. In this theorem, we require a condition on the Fourier expansion coefficient $f_{n}$ in (32). This condition is very difficult to check. To improve this, in the next theorem, we only require the assumption of $u$, not to depend on the function $f$.

Theorem 7. Let $g, g^{\epsilon}$ be as in Theorem 1. Assume that problem (1)-(4) has a solution $u$ such that

$$
\left\|u_{x}(\cdot, y)\right\|_{2}^{2}+\left\|u_{y}(\cdot, y)\right\|_{2}^{2}<\infty
$$

If we select $\alpha=\epsilon^{k}$ for $0<k<1$, then

$$
\left\|v^{\epsilon}(\cdot, y)-u(\cdot, y)\right\|_{2} \leq \epsilon^{1-k}+\frac{P}{2 k \ln (1 / \epsilon)}
$$

for every $y \in[0,1]$ where

$$
P=\sqrt{\left\|u_{x}(\cdot, y)\right\|_{2}^{2}+\left\|u_{y}(\cdot, y)\right\|_{2}^{2}} .
$$

Proof. Combining (15) and (20), we obtain

$$
\begin{aligned}
& u_{n}^{\epsilon}(y)-u_{n}(y) \\
& =\frac{-1}{2}\left(\frac{\alpha}{\alpha+e^{-n y}}\right)\left[e^{n y} g_{n}+\frac{1}{n} \int_{0}^{y} e^{n(y-s)} f_{n}(s) d s\right] \\
& =\frac{-1}{2}\left(\frac{\alpha}{\alpha+e^{-n y}}\right)\left[u_{n}(y)+\frac{1}{n} \frac{d}{d y} u_{n}(y)\right] \\
& =\frac{-1}{2}\left(\frac{\alpha}{\alpha n+n e^{-n y}}\right)\left[n u_{n}(y)+\frac{d}{d y} u_{n}(y)\right] .
\end{aligned}
$$

For $z>0$, we consider the function $\varphi(z)=1 /\left(\alpha z+e^{-z}\right)$. By taking the derivative of $\varphi$, we get

$$
\varphi^{\prime}(z)=-\frac{\alpha-e^{-z}}{\left(\alpha z+e^{-z}\right)^{2}} .
$$

The function $\varphi(z)$ attains maximum value at the $z_{0}$ such that $\varphi^{\prime}\left(z_{0}\right)=0$. It follows that $e^{z_{0}}=1 / \alpha$ or $z_{0}=\ln (1 / \alpha)$. Hence

$$
\begin{aligned}
\frac{1}{\alpha z+e^{-z}} & \leq \frac{1}{\alpha z_{0}+e^{-z_{0}}} \\
& =\frac{1}{\alpha \ln (1 / \alpha)+\alpha} \\
& \leq \frac{1}{\alpha \ln (1 / \alpha)}
\end{aligned}
$$


TABLE 1

\begin{tabular}{lc}
\hline & $\epsilon_{1}=10^{-1}$ \\
$y$ & $\left\|v^{\epsilon}-u\right\|_{2}$ \\
\hline 0 & 0.0053 \\
0.1 & 0.0285 \\
0.2 & 0.0019 \\
0.3 & 0.0309 \\
0.4 & 0.0722 \\
0.5 & 0.1245 \\
0.6 & 0.1914 \\
0.7 & 0.2769 \\
0.8 & 0.3865 \\
0.9 & 0.5268 \\
\hline
\end{tabular}

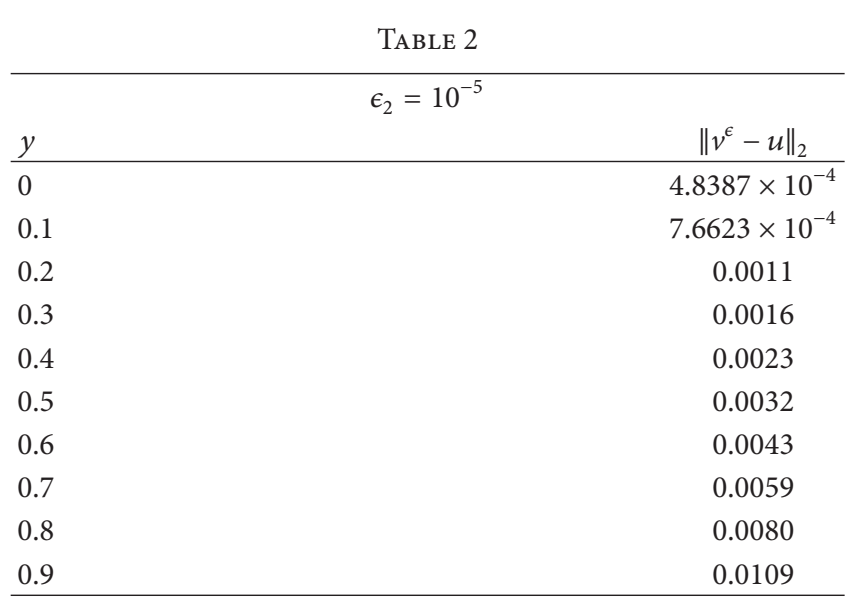

TABLE 3

\begin{tabular}{lc}
\hline & $\epsilon_{3}=10^{-10}$ \\
$y$ & $\left\|v^{\epsilon}-u\right\|_{2}$ \\
\hline 0 & $1.5665 \times 10^{-6}$ \\
0.1 & $2.4632 \times 10^{-6}$ \\
0.2 & $3.6404 \times 10^{-6}$ \\
0.3 & $5.1995 \times 10^{-6}$ \\
0.4 & $7.2769 \times 10^{-6}$ \\
0.5 & $1.0056 \times 10^{-5}$ \\
0.6 & $1.3786 \times 10^{-5}$ \\
0.7 & $1.8801 \times 10^{-5}$ \\
0.8 & $2.5551 \times 10^{-5}$ \\
0.9 & $3.4647 \times 10^{-5}$ \\
\hline
\end{tabular}

Using this inequality, we obtain

$$
\begin{aligned}
& \left\|u^{\epsilon}(\cdot, y)-u(\cdot, y)\right\|_{2}^{2} \\
& \quad=\frac{\pi}{2} \sum_{n=1}^{\infty}\left|u_{n}^{\epsilon}(y)-u_{n}(y)\right|^{2} \\
& \quad \leq \frac{\pi}{2} \sum_{n=1}^{\infty} \frac{1}{4}\left(\frac{\alpha}{\alpha n+e^{-n}}\right)^{2}\left[n u_{n}(y)+\frac{d}{d y} u_{n}(y)\right]^{2}
\end{aligned}
$$

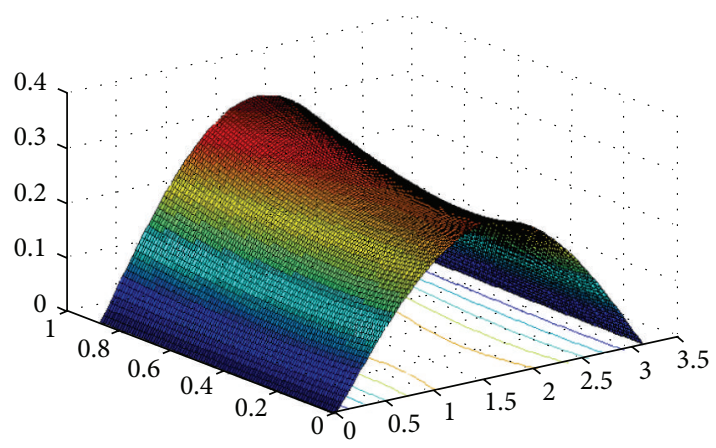

FIgURE 1: The exact solution in the case $0<y<1$.

$$
\begin{aligned}
& \leq \frac{1}{2 \ln ^{2}(1 / \alpha)} \frac{\pi}{2} \sum_{n=1}^{\infty}\left[n^{2}\left|u_{n}(y)\right|^{2}+\left|\frac{d}{d y} u_{n}(y)\right|^{2}\right] \\
& \leq \frac{1}{2 \ln ^{2}(1 / \alpha)}\left(\left\|u_{x}(\cdot, y)\right\|_{2}^{2}+\left\|u_{y}(\cdot, y)\right\|_{2}^{2}\right) .
\end{aligned}
$$

By Theorem 1 and $\alpha=\epsilon^{k}(0<k<1)$ and using the triangle inequality, we get

$$
\begin{aligned}
\left\|v^{\epsilon}(\cdot, y)-u(\cdot, y)\right\|_{2} \leq & \left\|v^{\epsilon}(\cdot, y)-u^{\epsilon}(\cdot, y)\right\|_{2} \\
& +\left\|u^{\epsilon}(\cdot, y)-u(\cdot, y)\right\|_{2} \\
& \leq \epsilon^{1-k}+\frac{P}{2 k \ln (1 / \epsilon)} .
\end{aligned}
$$

Remark 8. Condition (49) is natural and reasonable.

\section{A Numerical Experiment}

To illustrate the theoretical results obtained before, we will discuss the corresponding numerical aspects in this section. We consider a simple problem as follows:

$$
\begin{gathered}
\Delta u=\frac{3}{8}\left(e^{2 y}+e^{-2 y}\right) \sin x, \quad(x, y) \in(0, \pi) \times(0,1), \\
u(0, y)=u(\pi, y)=0, \\
u_{y}(x, 0)=0, \\
u(x, 0)=g(x) .
\end{gathered}
$$

Consider the exact data $g(x)=\sin x / 4$; then the exact solution to this problem is

$$
u(x, y)=\frac{e^{2 y}+e^{-2 y}}{8} \sin x .
$$




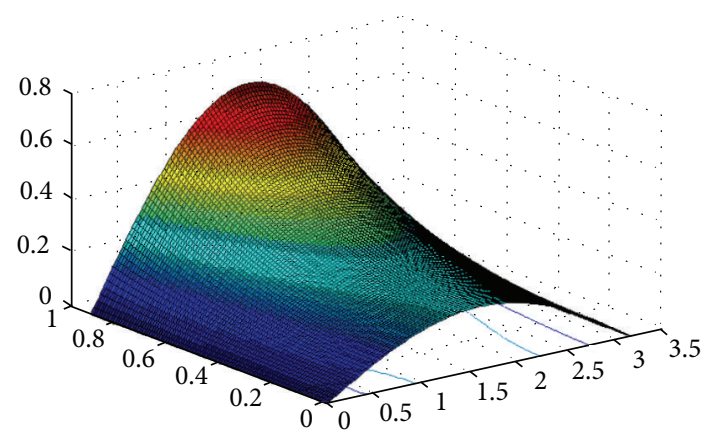

FIGURE 2: The regularized solution with $\epsilon_{1}=10^{-1}$.

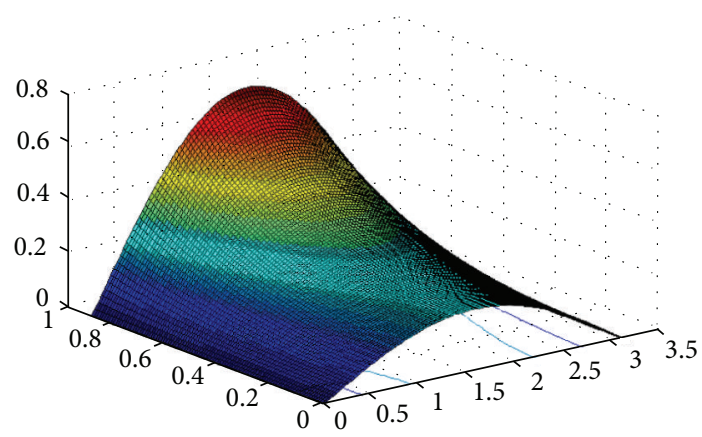

FIGURE 3: The regularized solution with $\epsilon_{2}=10^{-5}$.

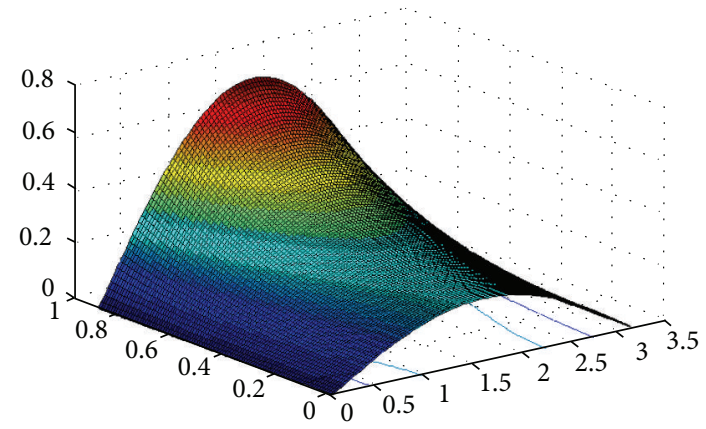

Figure 4: The regularized solution with $\epsilon_{3}=10^{-10}$.

Considering the measured data $g^{\epsilon}(x)=(\sqrt{32 / \pi} \epsilon+$ 1) $g(x)$, we have

$$
\begin{aligned}
\left\|g^{\epsilon}-g\right\|_{2} & =\left(\int_{0}^{\pi} \frac{32}{\pi} \epsilon^{2}(g(x))^{2} d x\right)^{1 / 2} \\
& =\left(\frac{2}{\pi} \epsilon^{2} \int_{0}^{\pi} \sin ^{2} x d x\right)=\epsilon .
\end{aligned}
$$

Let $\epsilon$ be $\epsilon_{1}=10^{-1}, \epsilon_{2}=10^{-5}, \epsilon_{3}=10^{-10}$, respectively. If we put

$$
y=\{0.1,0.2,0.3,0.4,0.5,0.6,0.7,0.8,0.9\}
$$

we get Tables 1, 2, and 3 for the case $0<y<1$ and we have the graphic that is displayed in Figures 1, 2, 3, and 4 on the interval $[0, \pi] \times[0,0.9]$.

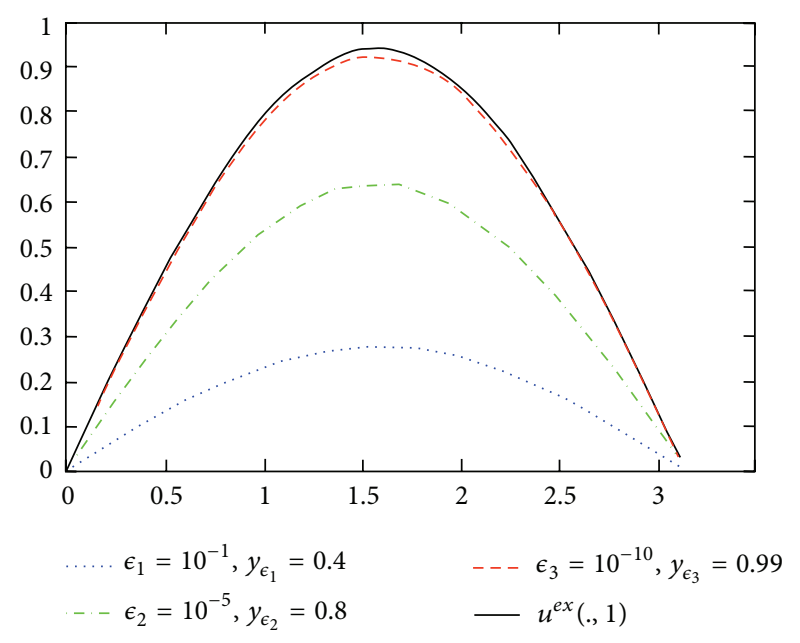

FIGURE 5: The exact solution and the regularized solution in the case $y=1$.

TABLE 4

\begin{tabular}{llc}
\hline & & $\left\|v^{\epsilon}\left(\cdot, y_{\epsilon}\right)-u(\cdot, 1)\right\|_{2}$ \\
\hline$\epsilon_{1}=10^{-1}$ & $y_{\epsilon_{1}}=0.4$ & 0.8319 \\
$\epsilon_{2}=10^{-5}$ & $y_{\epsilon_{2}}=0.8$ & 0.3792 \\
$\epsilon_{3}=10^{-10}$ & $y_{\epsilon_{3}}=0.99$ & 0.0225 \\
\hline
\end{tabular}

For each figure, we can find that the smaller the $\epsilon$ is, the better the computed approximation is. And the bigger the $y$ is, the worse the computed approximation is. Figure 5 shows the comparisons of the exact solution $u(x, y)$ and the approximation $v^{\varepsilon}(x, y)$ at the point $y=1$. In the case $y=1$, from (43) and using inequality $\ln (1-y)>-1 /(1-y)$ for every $0<y<1$, we get

$$
y_{\epsilon}>1-\frac{1}{\sqrt{2 \ln (1 / \epsilon)}} .
$$

Therefore, we will choose $y_{\epsilon_{1}}=0.4, y_{\epsilon_{2}}=0.8$, and $y_{\epsilon_{3}}=0.99$, with $\epsilon_{1}=10^{-1}, \epsilon_{2}=10^{-5}$, and $\epsilon_{3}=10^{-10}$, respectively. Numerical results are given in Table 4 .

\section{Conflict of Interests}

The authors declare that there is no conflict of interests regarding the publication of this paper.

\section{References}

[1] M. M. Lavrentev, V. G. Romanov, and S. P. Shishatskii, IllPosed Problems of Mathematical Physics and Analysis, vol. 64 of Translations of Mathematical Monographs, American Mathematical Society, Providence, RI, USA, 1986.

[2] R. Gorenflo, "Funktionentheoretische Bestimmung des Aussenfeldes zu einer zweidimensionalen magnetohydrostatischen Konfiguration," Zeitschrift für angewandte Mathematik und Physik, vol. 16, no. 2, pp. 279-290, 1965. 
[3] C. R. Johnson, "Computational and numerical methods for bioelectric field problems," Critical Reviews in Biomedical Engineering, vol. 25, p. 181, 1997.

[4] Z. Qian, C. Fu, and Z. Li, "Two regularization methods for a Cauchy problem for the Laplace equation," Journal of Mathematical Analysis and Applications, vol. 338, no. 1, pp. 479-489, 2008.

[5] D. E. Beskos, "Boundary element methods in dynamic analysis: part II (1986-1996)," Applied Mechanics Reviews, vol. 50, no. 3, pp. 149-197, 1997.

[6] I. Harari, P. E. Barbone, M. Slavutin, and R. Shalom, "Boundary infinite elements for the Helmholtz equation in exterior domains," International Journal for Numerical Methods in Engineering, vol. 41, no. 6, pp. 1105-1131, 1998.

[7] L. Marin, L. Elliott, P. J. Heggs, D. B. Ingham, D. Lesnic, and $\mathrm{X}$. Wen, "BEM solution for the Cauchy problem associated with Helmholtz-type equations by the Landweber method," Engineering Analysis with Boundary Elements, vol. 28, no. 9, pp. 1025-1034, 2004

[8] M. V. Klibanov and F. Santosa, "Computational quasi-reversibility method for Cauchy problems for Laplace's equation," SIAM Journal on Applied Mathematics, vol. 51, no. 6, pp. 1653$1675,1991$.

[9] A. Qian, X. Xiong, and Y. Wu, "On a quasi-reversibility regularization method for a Cauchy problem of the Helmholtz equation," Journal of Computational and Applied Mathematics, vol. 233, no. 8, pp. 1969-1979, 2010.

[10] N. H. Tuan, D. D. Trong, and P. H. Quan, "A new regularization method for a class of ill-posed Cauchy problems," Sarajevo Journal of Mathematics, vol. 6(19), no. 2, pp. 189-201, 2010.

[11] Z. Qian, C. Fu, and X. Xiong, "Fourth-order modified method for the Cauchy problem for the Laplace equation," Journal of Computational and Applied Mathematics, vol. 192, no. 2, pp. 205-218, 2006

[12] R. Shi, T. Wei, and H. H. Qin, "A fourth-order modified method for the Cauchy problem of the modified Helmholtz equation," Numerical Mathematics, vol. 2, pp. 326-340, 2009.

[13] C. Fu, X. Feng, and Z. Qian, "The Fourier regularization for solving the Cauchy problem for the Helmholtz equation," Applied Numerical Mathematics, vol. 59, no. 10, pp. 2625-2640, 2009.

[14] A. Qian, J. Mao, and L. Liu, "A spectral regularization method for a cauchy problem of the modified helmholtz equation," Boundary Value Problems, vol. 2010, Article ID 212056, 13 pages, 2010.

[15] N. H. Tuan, D. D. Trong, and P. H. Quan, "A note on a Cauchy problem for the Laplace equation: regularization and error estimates," Applied Mathematics and Computation, vol. 217, no. 7, pp. 2913-2922, 2010.

[16] Y. C. Hon and T. Wei, "Backus-Gilbert algorithm for the Cauchy problem of the Laplace equation," Inverse Problems, vol. 17, no. 2, pp. 261-271, 2001.

[17] L. Eldén and V. Simoncini, "A numerical solution of a Cauchy problem for an elliptic equation by Krylov subspaces," Inverse Problems, vol. 25, no. 6, Article ID 065002, 22 pages, 2009.

[18] L. Eldén and F. Berntsson, "A stability estimate for a Cauchy problem for an elliptic partial differential equation," Inverse Problems, vol. 21, no. 5, pp. 1643-1653, 2005.

[19] L. Marin and D. Lesnic, "The method of fundamental solutions for the Cauchy problem associated with two-dimensional Helmholtz-type equations," Computers and Structures, vol. 83, no. 4-5, pp. 267-278, 2007.
[20] H. H. Qin and T. Wei, "Modified regularization method for the Cauchy problem of the Helmholtz equation," Applied Mathematical Modelling, vol. 33, no. 5, pp. 2334-2348, 2009.

[21] T. Regińska and U. Tautenhahn, "Conditional stability estimates and regularization with applications to cauchy problems for the helmholtz equation," Numerical Functional Analysis and Optimization, vol. 30, no. 9-10, pp. 1065-1097, 2009.

[22] U. Tautenhahn, "Optimal stable solution of cauchy problems for elliptic equations," Zeitschrift fur Analysis und ihre Anwendung, vol. 15, no. 4, pp. 961-984, 1996.

[23] D. N. Hào, N. Van Duc, and D. A. Sahli, "A non-local boundary value problem method for the Cauchy problem for elliptic equations," Inverse Problems, vol. 25, no. 5, Article ID 055002, 2009.

[24] X. Feng, L. Eldén, and C. Fu, "A quasi-boundary-value method for the Cauchy problem for elliptic equations with nonhomogeneous Neumann data," Journal of Inverse and Ill-Posed Problems, vol. 18, no. 6, pp. 617-645, 2010.

[25] H. Cheng, X. Feng, and C. Fu, "A mollification regularization method for the cauchy problem of an elliptic equation in a multi-dimensional case," Inverse Problems in Science and Engineering, vol. 18, no. 7, pp. 971-982, 2010. 


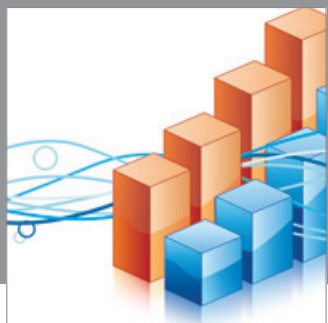

Advances in

Operations Research

mansans

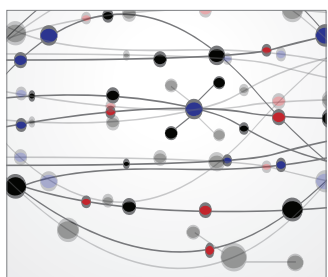

The Scientific World Journal
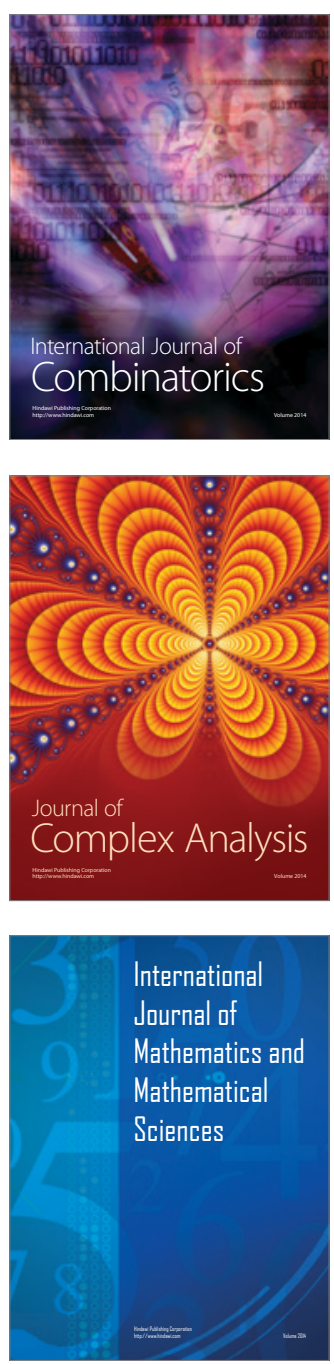
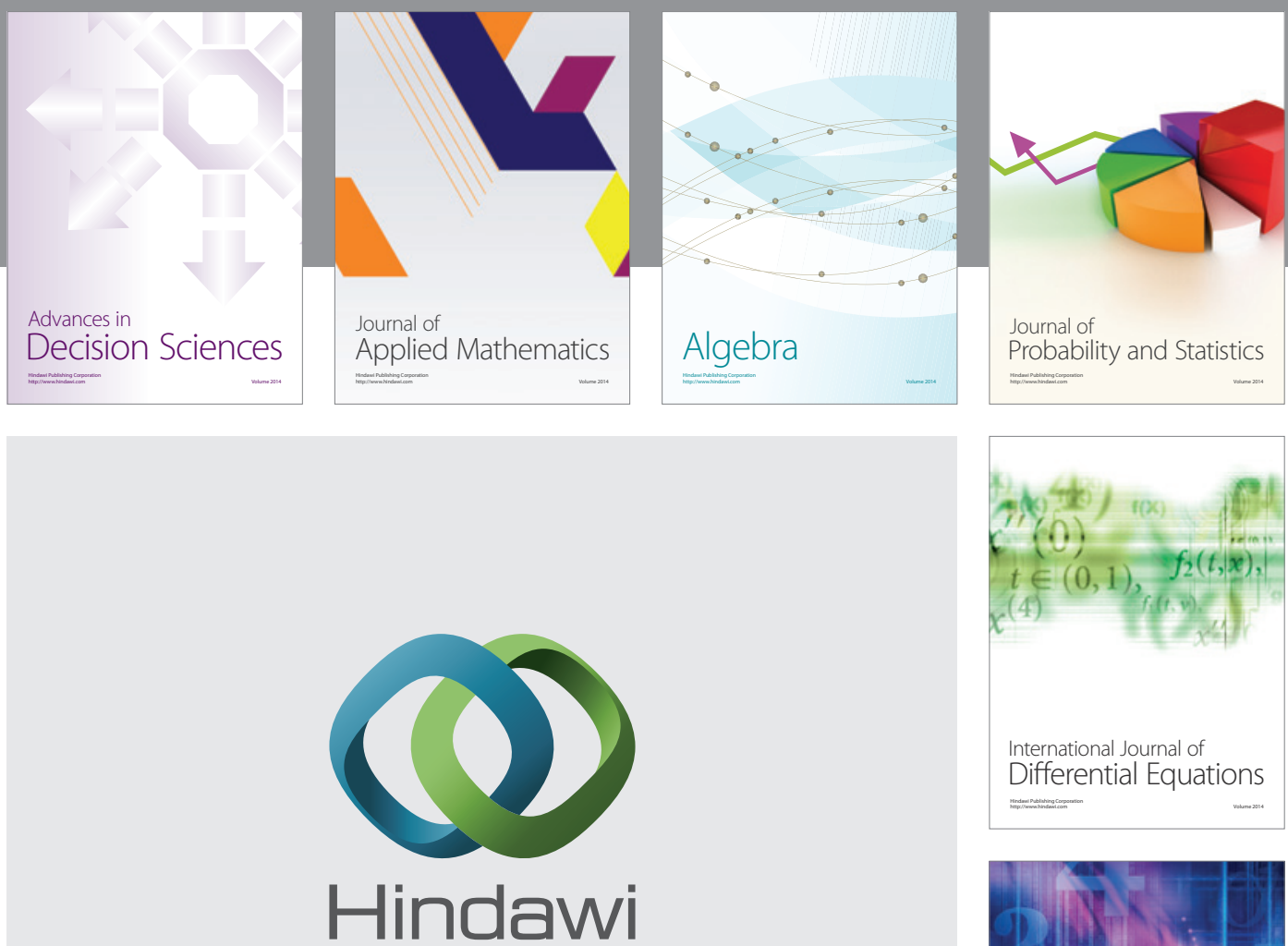

Submit your manuscripts at http://www.hindawi.com
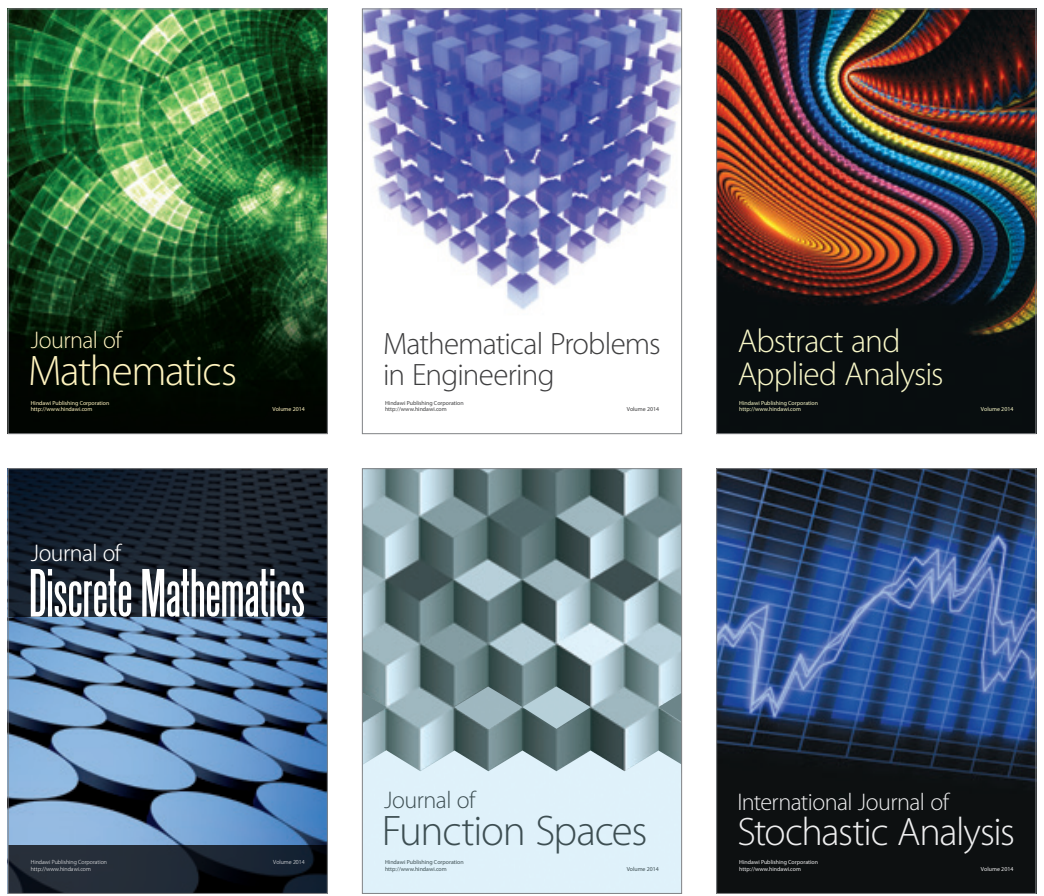

Journal of

Function Spaces

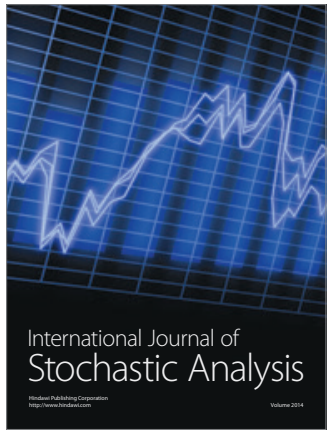

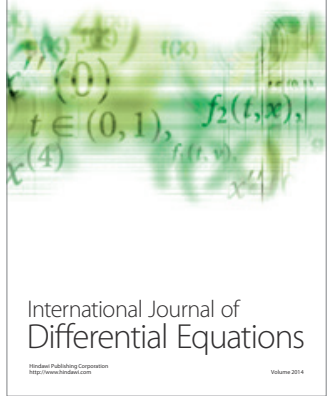
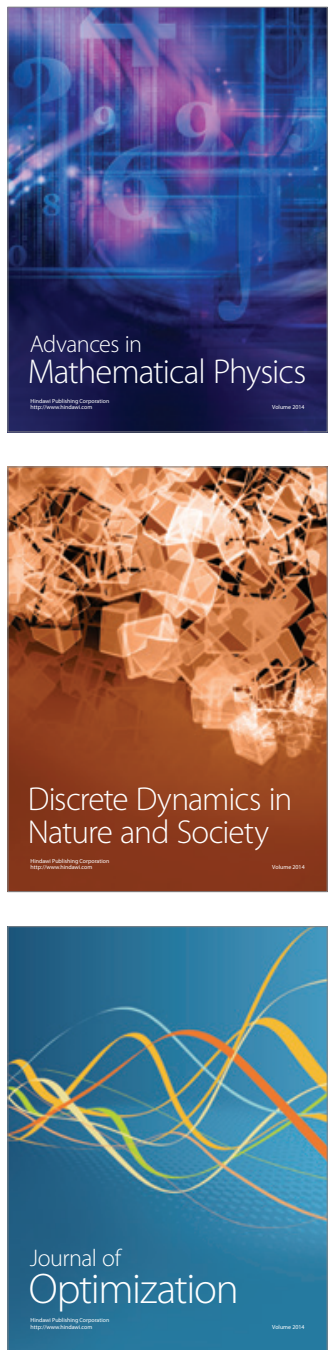